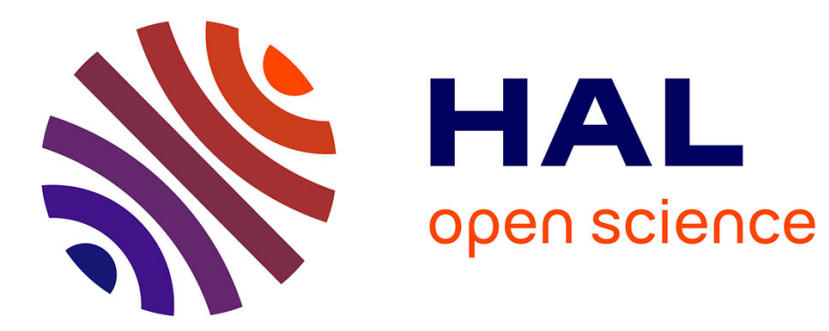

\title{
The flexible stem hypothesis: evidence from genetic data
} Jean-Michel Gibert

\section{To cite this version:}

Jean-Michel Gibert. The flexible stem hypothesis: evidence from genetic data. Development Genes and Evolution, 2017, 227 (5), pp.297-307. 10.1007/s00427-017-0589-0 . hal-01628015

\section{HAL Id: hal-01628015 https://hal.science/hal-01628015}

Submitted on 2 Nov 2017

HAL is a multi-disciplinary open access archive for the deposit and dissemination of scientific research documents, whether they are published or not. The documents may come from teaching and research institutions in France or abroad, or from public or private research centers.
L'archive ouverte pluridisciplinaire HAL, est destinée au dépôt et à la diffusion de documents scientifiques de niveau recherche, publiés ou non, émanant des établissements d'enseignement et de recherche français ou étrangers, des laboratoires publics ou privés. 
1 The flexible stem hypothesis: evidence from genetic data

2

3 Jean-Michel Gibert

4

5 Sorbonne Universités, UPMC Univ Paris 06, CNRS, Biologie du Développement

6 Paris Seine, Institut de Biologie Paris Seine (LBD-IBPS), 75005 Paris, France.

7

8 e-mail: jean-michl.gibert@upmc.fr

9 tel: 0033144275842

10 Orcid number: 0000-0002-1579-0266

11

12 Acknowledgments: I thank Sophie Gournet for the drawings illustrating this

13 review. I thank Frédérique Peronnet, Emmanuèle Mouchel-Vielh and Virginie

14 Courtier-Orgogozo for critical reading of the manuscript and stimulating

15 discussions. I thank the two anonymous reviewers for comments that 16 significantly enriched the manuscript.

17

18

19 
21 Abstract: Phenotypic plasticity, the ability of a given genotype to produce different phenotypes in response to distinct environmental conditions, is widely observed in the wild. It is believed to facilitate evolution and, under the "flexible stem hypothesis", it is thought that an ancestral plastic species can be at the origin of sister lineages with divergent phenotypes fixed by genetic assimilation of alternative morphs. We review here the genetic mechanisms underlying such phenomenon. We show several examples in which the same gene shows transcriptional plasticity in response to environmental factors and divergence of expression within or between species. Thus, the same gene is involved both in the plasticity of a trait and in the evolution of that trait. In a few cases, it can be traced down to cisregulatory variation in this gene and, in one case, in the very same regulatory sequence whose activity is modulated by the environment.

34 These data are compatible with the "flexible stem hypothesis" and also suggest that the evolution of the plasticity of a trait and the evolution of the trait are not completely uncoupled as they often involve the same locus. Furthermore, the "flexible stem hypothesis" implies that it is possible to canalize initially plastic phenotypes. Several studies have shown that it was possible through modification of chromatin regulation or hormonal signalling/response. Further studies of phenotypic plasticity in an evolutionary framework are needed to see how much the findings described in this review can be generalized. 
46 In developmental biology, the environment has long been thought to fulfil

47 essentially a permissive role, simply providing the required conditions for 48 normal development to occur. Thus, most developmental biology laboratories 49 still use standardized environmental conditions and focus on genetic factors to 50 dissect developmental mechanisms. However, in many cases the environment 51 has an instructive role, directing the development to particular alternative path 52 (Gilbert 2001). Phenotypic plasticity is a major concept which describes "the 53 property of a given genotype to produce different phenotypes in response to 54 distinct environmental conditions" (Pigliucci 2001). It is widely observed in the 55 wild, and this concept was formulated by Bradshaw in the sixties (Bradshaw 56 1965). Phenotypic plasticity can be an adaptation to fluctuating environments 57 such as seasonal variations, presence/absence of predators, or population 58 density. It can also be non adaptive and Schmalhausen used the term "morphose" 59 to describe aberrant phenotypes induced by extreme environmental conditions 60 (Schmalhausen 1949). A major tool to illustrate phenotypic plasticity is the 61 reaction norm, first drawn by Woltereck in 1909 (Woltereck 1909), which 62 represents the value of a quantitative phenotype produced by a given genotype 63 in function of an environmental parameter. Note that "genotype" and "phenotype" 64 were defined only shortly after by Johannsen (1911) who reinterpreted the 65 reaction norms of Woltereck (Johannsen 1911). Indeed, Woltereck observed 66 that different pure lines could produce different morphologies in a given 67 environment but the same morphology in another environment. He interpreted 68 this as inconsistent with the existence of constant differences between the 69 genotypes. Because he did not distinguish between genotypes and phenotypes, 
he thought that the different pure lines had the same genotype in a given

71 environment (when they had the same phenotype) and different genotypes in

72 another environment (when they had different phenotypes). When phenotypic

73 plasticity leads to discrete morphs, these morphs are called polyphenisms.

74 Phenotypic plasticity can generate, within a single species, morphological

75 differences of a magnitude usually observed between distantly related species.

76 As selection operates on phenotypes, this direct input of the environment on the

77 expression of the genotype must be taken into account to understand

78 evolutionary processes. This was clearly formulated by Schmalhausen (1949)

79 (Schmalhausen 1949): "Darwin has previously noted the fundamental importance

80 of inherited variability (mutations) for the evolution of organisms. However,

81 mutations have different expressions under diverse conditions. If natural selection

82 is the basic factor in evolution, then the concrete expression of individual

83 characteristics under given environmental conditions is of decisive importance in

84 this process ». Thus, the role of phenotypic plasticity in evolution has been

85 actively discussed (Pfennig et al. 2010; Moczek et al. 2011; Laland et al. 2014,

86 2015; Schneider and Meyer 2017). The issue is complex and certain empirical

87 results suggest that it is important to distinguish between adaptive and non-

88 adaptive plasticity as they may have opposite effects on genetic divergence

89 (Ghalambor et al. 2015).

90 The idea that an ancestral plastic species could have been at the origin of

91 divergent lineages with pronounced phenotypic differences has been proposed

92 by Mary-Jane West-Eberhard as the "flexible stem hypothesis" (West-Eberhard

93 2003). It is intimately linked to the process of genetic assimilation discovered by

94 Conrad Waddington more than 50 years ago (Waddington 1952, 1956, 1959). In 
95 his experiments on Drosophila, Waddington showed that a phenotype initially

96 induced by the environment can be fixed by selection and become constitutively

97 expressed even in the absence of the initial environmental factor. Most of these

98 experiments were based on developmental defects induced by stressors such as

99 heatshock (crossveinless phenotypes on wings) (Waddington 1952) or ether

100 vapour (bithorax phenocopies) (Waddington 1956). However, they could be

101 generalized to adaptive phenotypes and had therefore major evolutionary

102 implications. Indeed, in another Waddington experiment, the morphological

103 change induced by the environment, i.e. size increase of the anal papilla of larvae

104 in response to high salt concentration, was likely adaptive and was also

105 genetically assimilated (Waddington 1959). Importantly, genetic assimilation is a

106 genuine evolutionary process based on selection of genetic variation existing in

107 the population submitted to environmental changes. Indeed, Waddington

108 experiments were later repeated by Bateman on outbred or isogenized stocks

109 and it was shown that genetic assimilation did not happen in the absence of

110 genetic variation in the population (Dworkin 2005). This genetic variation is

111 cryptic in the original environment in which it accumulates as it has no

112 phenotypic consequences. This genetic variation translates into phenotypic

113 variation in the new environment, which allows genetic assimilation by

114 selection. Thus, a lineage characterized by a constitutively expressed morph

115 could evolve from a plastic ancestor by genetic assimilation of a phenotype

116 initially induced by environmental change.

117 This has lead Mary Jane West-Eberhard to propose that genes act more as

118 followers than as leaders during evolution (West-Eberhard 2005). Although very

119 stimulating, this idea cannot be generalized to the evolution of all traits. Indeed, 
in many examples, genetic polymorphisms have played a major role in the origin

121 of a phenotype later fixed during evolution (Schwander and Leimar 2011). For

122 example, a particular allele of the pigmentation gene tan leading to lighter

123 pigmentation is segregating in Drosophila americana and has been fixed in its

124 sister species Drosophila novamexicana which has a derived pale phenotype

125 (Wittkopp et al. 2009). However, although the "plasticity first hypothesis" does

126 not apply to all traits, it is worth investigating it empirically. The flexible stem

127 model of adaptive radiation predicts that the pattern of phenotypic divergence

128 between derived lineages should mirror the pattern of developmental plasticity

129 in their common ancestor (Wund et al. 2008). Several studies have provided

130 empirical morphological data strongly suggesting that it could occur in nature

131 (Wund et al. 2008). For example, the pharyngeal jaws of midas cichlids show a

132 morphological plasticity in response to the hardness of the diet which parallels

133 the morphological diversity observed between specialised species (Muschick et

134 al. 2011). Similarly, stress-induced changes in mandible shape in Sorex shrews

135 correspond to traits showing divergence between species (Badyaev and

136 Foresman 2000).

137 What we would like to review and discuss in this article is the underlying genetic

138 bases of the flexible stem hypothesis. Indeed, under this hypothesis, it is 139 expected that the genetic mechanisms that generate the plastic phenotypes 140 should be the same as those that generate the phenotypic divergence. The idea 141 that phenotypic plasticity is under genetic control was initially developed by 142 Bradshaw (Bradshaw 1965) and a recurrent question was the existence and the 143 nature of "plasticity genes" (Via et al. 1995). However, Via considered that 144 modifications of reaction norms was an indirect consequence of the selection on 
145 the mean phenotype in each separate environment and that no plasticity genes

146 were required (Via 1993). Scheiner, Schlichting and Pigliucci objected to Via that

147 the plasticity of a trait could evolve independently of the mean of the trait

148 because the plasticity and the mean of the trait are under the control of different 149 genes (Scheiner 1993; Schlichting and Pigliucci 1993). This debate settled down 150 in the mid nineties in favour of the existence of plasticity genes. Two kinds of 151 genetic mechanisms involved in phenotypic plasticity were proposed (Via et al. 152 1995). The first one, "allelic sensitivity", refers to alleles expressed differently in 153 distinct environment. This could correspond to a single gene showing 154 transcriptional plasticity or to an enzyme active in only some environmental 155 conditions for example. The second genetic mechanism, "gene regulation", 156 corresponds to a regulatory switch under environmental control, which would 157 lead to the activation of a panel of target genes depending on the environment. 158 Obviously, the two mechanisms may blur as the regulatory locus influences the 159 amount of products of its targets, thus mediating allelic sensitivity (Via et al. 160 1995). Clearly, a great deal of information could be gained by genetic dissection 161 of phenotypic plasticity in a variety of organisms.

162 A few empirical studies have identified genes involved in the phenotypic 163 plasticity of particular traits and in several cases, data are available regarding 164 genes involved in the variation within or between species of the traits of interest. 165 Thus, it is possible to analyse the genetic changes that underlie genetic 166 assimilation of initially plastic traits. Furthermore, several studies have dissected 167 the mechanisms involved in phenotypic plasticity and environmental 168 canalization. The typical questions we would like to address are the following: 169 What are the relationships between genes involved in phenotypic plasticity of a 
trait and genes involved in the evolution of this trait? What kind of mutations are

171 selected during the evolution of these traits? Are they regulatory or coding

172 mutations? Do they target cis-regulatory sequences of a modulated gene or trans-

173 acting factors? How is it possible to make independent of the environment an

174 initially plastic trait? We present here several examples of phenotypic plasticity,

175 chosen in animals or plants, whose genetic bases have been addressed through

176 candidate gene approach or global transcriptome analyses. Most of these

177 analyses are performed in an evolutionary context (either artificial or natural) or

178 can be complemented by other studies on the evolution of the particular traits of

179 interest in closely related species. Furthermore, we describe studies dissecting

180 the mechanisms involved in phenotypic plasticity and environmental 181 canalisation. Modulation of such mechanisms must be involved in the 182 environmental canalization of initially plastic traits involved in the flexible stem 183 hypothesis.

\section{Ether-induced Ultrabithorax phenocopies in Drosophila}

186 Treatment of Drosophila embryos with ether vapor leads to phenocopies of the 187 bithorax mutant in adults (Waddington 1956). This corresponds to partial 188 homeotic transformation of the third thoracic segment into the second thoracic 189 segment. In Drosophila, like in all dipterans, the dorsal appendages of the third 190 thoracic segments are not wings but little round structures named halteres. In 191 bithorax phenocopies, halteres are partially transformed into wings and the 192 postnotum is partially transformed into an additional mesonotum (Figure 1).

193 Waddington showed that this ether-induced phenotype can be genetically 194 assimilated (Waddington 1956). By selection after ether treatment at each 
195 generation, he could progressively increase the proportion of individual showing

196 the bithorax phenocopy. At the eighth generation, this phenotype was observed 197 in some individuals even in the absence of ether treatment (Figure 1). The 198 bithorax phenotype was therefore genetically assimilated. Waddington 199 experiments were repeated by Gibson and Hogness (Gibson and Hogness 1996). 200 They analysed the expression of the homeotic gene Ultrabithorax (Ubx) which is 201 expressed in haltere but not wing imaginal disc and is responsible for the 202 different identities of these organs. They showed that ether-induced bithorax 203 phenocopies are caused by clonal loss of expression of $U b x$ in the haltere 204 imaginal disc. Furthermore, using polymorphism in $U b x$, they could show that 205 the frequency of particular alleles of $U b x$ increased during genetic assimilation of 206 the bithorax phenotype. The Ubx polymorphism was not the only source of 207 genetic variation affecting sensitivity to ether, but it had a major effect. Thus, $U b x$ 208 expression is sensitive to ether and during genetic assimilation of this 209 phenotype, artificial selection targeted the $U b x$ locus itself.

\section{Transcriptional response to osmotic challenge in killifish:}

212 The Atlantic killifish (Fundulus heteroclitus) can be found along a steep salinity 213 gradient in Atlantic coast estuaries and can quickly adjust to extremes in 214 environmental salinity (Figure 2). Thus, this species is able to occupy an 215 extremely wide osmotic niche. Hypo-osmotic challenge induces a change of 216 cellular morphology of the apical surface of the gill epithelium (Whitehead et al. 217 2011) (Figure 2). In sea water, the apical surface of the gill epithelium is made of 218 pavement cells interspersed with mitochondrion-rich cells possessing apical 219 crypt with small surface area (Whitehead et al. 2011). Exposure to fresh water 
leads to enlargement of the apical surface of mitochondrion-rich cells. This

221 modification occurs within a few days, but its speed and the precise morphology

222 of the gill epithelium differs between populations originating from different

223 point of the salinity gradient. Analysis of the transcriptome of gills in fish

224 submitted to hypo-osmotic challenge allowed to identify sets of genes whose

225 expression was modulated by salinity (Whitehead et al. 2011). It was found that

226 genes showing transcriptional plasticity during osmotic challenge are more

227 likely to show divergence of expression between populations living in different

228 environment (Whitehead et al. 2011, 2012). Indeed, many of the genes

229 modulated during hypo-osmotic challenge show adaptive divergence in

230 expression in populations living exclusively in freshwater habitat. Among these

231 genes, many have a well known role in osmotic regulation, for example

232 Aquaporin 3 (AQP3), the sodium/potassium transporting ATPAse ATP1A1 or 233 versican (VCAN) (Whitehead et al. 2012).

234

235 Plasticity of wing eyespots in the butterfly Bicyclus anynana

236 The African satyrine butterfly Bicyclus anynana shows seasonal polyphenisms. In

237 the wet season morph, conspicuous eyespots made of concentric rings of

238 differently pigmented scales are observed on the ventral wings. In the dry season

239 morph, the ventral eyespots are absent. Only a reduced version of the white spot

240 in the centre of the eyespot is visible. The wet and dry season morphs can be

241 produced in the laboratory by rearing the larvae at $27^{\circ} \mathrm{C}$ or $17^{\circ} \mathrm{C}$ respectively

242 (Brakefield et al. 1996) (Figure 3).

243 Many developmental regulatory genes are expressed in developing eyespots of

244 Bicyclus anynana, among which Distal-less (DIl) which is expressed in the future 
245 centre of the eyespot (Brakefield et al. 1996; Shirai et al. 2012). This is not

246 simply a correlation as it was recently shown by gain and loss of function

247 experiments using transgenic Bicyclus anynana that Dll is involved in eyespot

248 formation (Monteiro et al. 2013). Indeed, overexpression of Dll increases eyespot

249 size and induces ectopic eyespots. Conversely, decreasing Dll expression reduces

250 eyespot size. Furthermore, the expression of $D l l$ in presumptive eyespots is

251 modulated by temperature: it is stronger and wider in pupae grown at $27^{\circ} \mathrm{C}$ that

252 will produce the wet season morph (Brakefield et al. 1996). Thus, Dll is one of

253 the genes involved in eyespot plasticity.

254 It is possible to fix alternative morphs by artificial selection performed at

255 intermediate temperature. This lead to a LOW line and a HIGH line showing 256 almost non plastic dry season and wet season phenotypes, respectively

257 (Brakefield et al. 1996) (Figure 3). Thus, the wet or dry season morphs can be 258 genetically assimilated and become virtually independent of the rearing 259 temperature. This correlates with a modification of Dll expression, which is 260 stronger and wider in pupae of the HIGH line than in pupae of the LOW line 261 grown in the same environmental conditions. Thus, genetic assimilation of the 262 alternative seasonal morphs has involved modulation of Dll expression. Whether 263 this genetic assimilation was caused by selection at the Dll locus itself (cis 264 variation) or by trans-acting factors was not tested in this experiment. However, 265 another selection experiment targeting the size of dorsal eyespots (which 266 however are not plastic), showed that natural genetic variation in Dll was linked 267 to eyespot size variation in Bicyclus anynana (Beldade et al. 2002). Thus, 268 variation at the Dll locus with significant impact on eyespot patterning exists in 269 Bicyclus anynana. 


\section{Polyphenism of cuticular teeth in diplogastrid nematods}

272 The diplogastrid nematod Pristionchus pacificus displays two alternative tooth 273 morphs, stenostomatous (St) or eurystomatous (Eu), that are produced after an 274 irreversible decision during larval development (Bento et al. 2010) (Figure 4).

275 Eu morph is adapted to predation on other nematods, whereas St morph is 276 adapted to bacterial diet. The ratio of individuals with St or Eu teeth varies 277 between strains but is also influenced by environmental factors such as 278 crowding and starvation (Bento et al. 2010; Ragsdale et al. 2013). The high 279 expression of the gene eud-1, encoding a sulfatase, is necessary and sufficient to 280 produce the Eu phenotype in Pristionchus pacificus (Ragsdale et al. 2013). Loss of 281 function mutations of eud-1 are dominant and lead to the St phenotype. 282 Difference of the Eu/St ratio between wild isolates of Pristonchus pacificus 283 correlates with difference in eud-1 expression. Furthermore, eud-1 over284 expression of is sufficient to transform high St strains into Eu ones. The role of 285 eud-1 in tooth dimorphism extends to the other diplogastrid species Pristionchus 286 exspectacus (Ragsdale et al. 2013). Indeed, through hybrid crosses between 287 various $P$. pacificus lines and a $P$. exspectacus St line, it was shown that the St 288 phenotype of the P. exspectatus line was caused by a low expression of eud-1. As 289 transformation of $P$. pacificus eud-1 mutant with the eud-1 allele of the $P$. 290 exspectatus St line leads to a Eu phenotype, the eud-1 allele of $P$. exspectacus is 291 functional and differences of eud-1 expression between $P$. pacificus and $P$. 292 exspectacus are caused by differences in trans-acting factors. Thus, divergence in 293 the ratio of tooth morphs might have involved several components of the eud-1 294 network. 
295 It is thought that the stem species in diplogastrid nematods was polyphenic 296 (Kiontke and Fitch 2010). Many descendant species such as Pristionchus pacificus 297 have kept both morphs, but others have become exclusively Eu or St. 298 Interestingly, recent morphometric analysis of diplogastrid mouth structures in 299 a more global evolutionary framework revealed a rapid process of diversification 300 associated with developmental plasticity (Susoy et al. 2015): The gain of 301 dimorphic mouth structures was associated with an increase rate of evolution 302 and the appearance of numerous new species with diversified mouth parts.

303

\section{Modulation of leaf margin dissection by temperature in Capsella}

305 The two brassicaceae sister species Capsella rubella and Capsella grandiflora 306 show differences in leaf margin dissection (Sicard et al. 2014). Leaf margin 307 dissection is more pronounced in Capsella rubella and is increased at low 308 temperature $\left(16^{\circ} \mathrm{C}\right.$ versus $\left.22^{\circ} \mathrm{C}\right)$. It was shown that the major locus responsible

309 for the difference in leaf margin dissection between C. rubella and C. grandiflora 310 is the homeobox gene $R C O-A$ which is expressed in sinuses between growing 311 lobes of developing leaves in C. rubella (Sicard et al. 2014). This difference of 312 expression level is caused by insertions in the $C$. rubella $R C O-A$ promoter. The 313 effect of the $R C O-A$ region on leaf margin dissection is visible in nearly isogenic 314 lines differing only in the region of $R C O-A$, NILgg (carrying the $R C O-A$ allele of $C$. 315 grandiflora) and NILrr (carrying the RCO-A allele of $C$. rubella) (Figure 5). In 316 addition, $R C O-A$ expression is strongly modulated by temperature. Expression of 317 the C. grandiflora $R C O-A$ allele is extremely weak at $22^{\circ} \mathrm{C}$ and the leaves show no 318 dissection. This allele is up-regulated more than 150 folds at $16^{\circ} \mathrm{C}$ compared to $31922^{\circ} \mathrm{C}$, which leads to leaf margin dissection. The C. rubella RCO-A allele is more 
expressed than the C. grandiflora one at $22^{\circ} \mathrm{C}$ and is upregulated less than 5 folds

321 between $22^{\circ} \mathrm{C}$ and $16^{\circ} \mathrm{C}$. Consequently, it leads to leaf margin dissection at both

322 temperatures in C. rubella.

323 In another brassicae species, Arabidopsis thaliana, the $R C O-A$ locus is deleted and

324 there is no leaf margin dissection. Insertion of transgenes carrying Capsella

325 rubella $R C O-A$ gene in Arabidopsis thaliana is sufficient to induce leaf margin

326 dissection (Sicard et al. 2014).

327 Thus, the same locus is directly involved in phenotypic plasticity and species

328 divergence regarding leaf margin dissection. However, as the activity of the

329 regulatory sequences of $R C O-A$ responsible for the difference of expression

330 between the two Capsella species was not studied with a reporter gene in 331 transgenic lines grown at different temperatures, it is not known whether they 332 also mediate the effect of the environment.

334 Thermal plasticity of abdominal pigmentation in Drosophila melanogaster 335 females

336 Cold induces melanism in many insects (Gibert et al. 2000; Michie et al. 2010;

337 Fedorka et al. 2013). This is thought to be beneficial as dark pigmentation 338 increases body temperature. However, pigmentation is highly pleiotropic and is 339 also correlated to many other traits such as longevity, fecundity or immunity 340 (Wittkopp and Beldade 2009; Fedorka et al. 2013; Kutch et al. 2014; Rajpurohit 341 et al. 2016). In Drosophila melanogaster females, abdominal pigmentation is 342 strongly modulated by temperature (Figure 6). This is particularly pronounced 343 in the more posterior segments (A5, A6 and A7) (Gibert et al. 2000). 344 Correspondingly, the expression of several genes encoding the enzymes of the 
345 cuticular pigment synthesis pathway in the abdominal epidermes of late pupae 346 and young adults is modulated by temperature (Gibert et al. 2016, 2017). 347 However, among them, the gene tan stands out, being expressed seven times 348 more in the abdominal epidermis of young females grown at $18^{\circ} \mathrm{C}$ than $29^{\circ} \mathrm{C}$. The 349 activity of tan increases melanin production and genetic experiments showed 350 that temperature modulation of tan expression plays an essential role in thermal 351 plasticity of female abdominal pigmentation (Gibert et al. 2016). Furthermore, 352 temperature modulates the activity of the tan abdominal enhancer $\left(t_{-} M S E\right)$ and 353 the level of the active histone mark H3K4me3 on tan promoter. Interestingly, tan 354 is well known for its involvement in pigmentation variation and divergence in 355 the Drosophila melanogaster species subgroup. In Drosophila melanogaster, 356 independent genome wide association studies performed on European, African 357 and American populations have identified single nucleotide polymorphisms in 358 the $t_{-} M S E$ involved in variation of female abdominal pigmentation (Bastide et al. 359 2013; Dembeck et al. 2015; Endler et al. 2015). In Drosophila erecta, female 360 dimorphic pigmentation is caused by genetic variation in the $t_{-} M S E$ maintained 361 by balancing selection (Yassin et al. 2016). In Drosophila santomea, several 362 independent loss of function mutations located in the $t_{-} M S E$ have lead to the light 363 phenotype of this species, whereas in the sister species Drosophila yakuba a 364 functional $t$-MSE enhancer is associated with dark abdominal pigmentation 365 (Jeong et al. 2008). Thus, in the Drosophila melanogaster species subgroup, 366 plasticity and variation between species of abdominal pigmentation work 367 through modulation of the same gene and involves genetic changes in the very 368 same regulatory sequence, $t_{-} M S E$, that also mediates the effect of temperature on 369 tan expression in Drosophila melanogaster. 


\section{Discussion}

372 These examples demonstrate that several genes showing transcriptional

373 plasticity in response to environmental changes are differentially expressed 374 within and/or between species and contribute to phenotypic variation and/or

375 divergence. Thus, the same genes respond to the environment and are involved 376 in evolution. It might seem predictable that the same genes are involved in the 377 plasticity of a trait and the variation/evolution of this trait. However, this is not 378 so obvious. Indeed, the same phenotypes can sometimes be produced by 379 different mechanisms. For example, Drosophila wing size can be modulated by 380 both temperature and clinal genetic variation. However, this occurs through 381 distinct mechanisms: temperature modulates cell size (Azevedo et al. 2002), 382 whereas clinal genetic variation primarily affects cell number (James et al. 1995). 383 Similar pigmentation phenotypes can be produced by modulating the expression 384 of different pigmentation genes (Wittkopp et al. 2003). For example, melanin 385 production can be increased by up-regulating tan or down-regulating ebony, that 386 encode enzymes with antagonist activities (True et al. 2005).

387 In the case of $R C O-A$ and tan, the variation or divergence in expression is caused 388 by mutations in the cis-regulatory sequences of the genes. This is also likely the 389 case for Ubx during genetic assimilation of the bithorax phenotype. Remarkably, 390 for tan, the same cis-regulatory sequence mediates, at least partly, the effect of 391 temperature. It is generally thought that cis-regulatory mutations are less 392 pleiotropic than mutations in trans-acting factors (Prud'homme et al. 2007). 393 Thus, targeting tan, a gene encoding a pigmentation enzyme, or $R C O-A$, whose 394 expression is limited to the sinuses between growing leaf lobes, limit potential 
deleterious effects. The recurrent involvement of $R C O-A$ and tan in evolution lead to their characterization as evolutionary hotspots (Martin and Orgogozo 2013;

397 Sicard et al. 2014). Why are these two genes targets of recurrent evolution ? For 398 a given allele, temperature sensitivity of $R C O-A$ and tan expression leads to 399 different phenotypes in different environments. Reciprocally, different alleles 400 will induce different phenotypes in the same environment. It follows that only 401 some genotypes will produce an optimal phenotype in a given environment. This 402 may lead to selection of different alleles of these genes in different environments 403 to reach an optimal phenotype. This is in particular the case if the phenotype 404 produced by the plastic response is not well adjusted to the environment. 405 Genetic variation will be selected to reduce or to increase the effect of the 406 environment. As the environment fluctuates spatially and temporally, adjusting 407 selection increases genetic variation with functional consequences at these loci. 408 This higher genetic variation facilitates the involvement of those loci in 409 evolution.

410 In the other cases presented in this review, the respective contributions of cis 411 and trans variations are not known (eud-1 in P. pacificus, genes modulated by 412 osmotic challenge in Fundulus, Dll in Bicyclus anynana) but the recent 413 identification of trans-regulators of eud-1 is a step towards answering this 414 question (Serobyan et al. 2016). However, what is clear is that evolution has 415 targeted components of the gene networks regulating the transcriptionally 416 plastic genes if not these genes themself. In most cases described in this review, 417 the gene showing transcriptional plasticity also exhibits genetic variation that 418 provides substrate for evolution through selection or simply drift. The fact that 419 the same networks, or even the same genes, are involved in plasticity and 
evolution contradicts somehow Scheiner's ideas on the decoupling of the genetic

421 processes controlling the plasticity and the mean of a trait (Scheiner 1993).

422 In addition, the "flexible stem hypothesis" implies that it is mechanistically

423 possible for a trait initially plastic to undergo environmental canalization and

424 become independent of the environment. Several studies have dissected the

425 mechanisms behind phenotypic plasticity and environmental canalization. Two

426 major mechanisms have been identified: regulation of chromatin structure and

427 hormonal signalling/response. It is known that environmental conditions can

428 modulate DNA methylation (Kucharski et al. 2008), histone post-translational

429 modifications (Simola et al. 2016; Gibert et al. 2016) or chromatin compaction

430 (Leung et al. 2014). Thus, stabilization of chromatin structure in an initially

431 plastic species, could lead to production of a constant phenotype independent of

432 the environment. For example, artificial down-regulation of DNA methylation in

433 honey-bees lead to the production of a queen phenotype from larvae that should

434 have developed as workers because on the diet they fed on (Kucharski et al.

435 2008). Some alleles might carry variation in their cis-regulatory sequences that

436 promotes the formation of a particular chromatin structure independently of the

437 environment. This might be the case for tan or $U b x$ and selection on this

438 variation might contribute to modify the sensitivity of their expression to the

439 environment. Regarding, hormonal regulation, it is known that differences of

440 plasticity between genotypes could be linked to differences of hormonal

441 signalling or response (Suzuki and Nijhout 2006). In Manduca sexta larvae,

442 artificial selection on temperature induced pigmentation variation lead to the

443 production of a polyphenic and a monophenic lines differing by their juvenile

444 hormone titer and response (Suzuki and Nijhout 2006). Interestingly a similar 
445 process applies to differences of plasticity between organs. In Drosophila, the

446 size of late larval brain and adult male genitalia is buffered against nutrient 447 restriction, whereas the size of other organs decreases under these conditions. 448 The buffering of genitalia size is caused by repression of FOXO expression in the 449 genital disc which makes it insensitive to the nutritional information provided by 450 insulin signalling (Tang et al. 2011). The buffering of late larval brain size is 451 caused by cell-type specific expression of Alk and its ligand Jelly Belly which 452 renders neuroblasts insensitive to amino acid and insulin sensing (Cheng et al. 453 2011). Thus, by modifying the expression of key genes involved in hormonal 454 signalling or response it is possible to reduce or even abolish the influence of the 455 environment on a given trait. These studies illustrate what kind of mechanisms 456 might be involved in the genetic assimilation taking place during the formation 457 of a monophenic lineage under the "flexible stem hypothesis".

458 The examples described in this review are compatible with the "flexible stem 459 hypothesis" (West-Eberhard 2003), which postulate ancestral plasticity followed 460 by genetic assimilation of alternative morphs in derived lineages. Analysis of 461 phenotypic plasticity of nematod feeding structures in an evolutionary 462 framework supports indeed that plasticity was ancestral and that one morph 463 was stabilized (genetically assimilated) in particular lineages whereas others 464 have kept plasticity (Kiontke and Fitch 2010). A definite demonstration of the 465 "flexible stem hypothesis" would be to follow allelic frequencies during genetic 466 assimilation of a phenotype of interest. This was done in only one of the 467 examples, but for a deleterious phenotype normally not observed in the wild, i.e. 468 the bithorax phenocopies (Gibson and Hogness 1996). Thus, more studies on the 469 genetic bases of phenotypic plasticity in an evolutionary conceptual framework 
are needed. In particular, it would be interesting to know whether the example

471 of tan and its $t_{-} M S E$ is just a particular case or whether it is predictive of a more 472 systematic involvement of the same regulatory sequences in phenotypic 473 plasticity and species variation and divergence.

References:

478

479 480

481 482 483 484

485

486

487

488

489

490

491

492

493

494 495 496

497 498

499

500

501
Azevedo RBR, French V, Partridge L (2002) Temperature modulates epidermal cell size in Drosophila melanogaster. J Insect Physiol 48:231-237.

Badyaev AV, Foresman KR (2000) Extreme environmental change and evolution: stress-induced morphological variation is strongly concordant with patterns of evolutionary divergence in shrew mandibles. Proc Biol Sci 267:371-7.

Bastide H, Betancourt A, Nolte V, et al (2013) A Genome-Wide, Fine-Scale Map of Natural Pigmentation Variation in Drosophila melanogaster. PLoS Genet 9:e1003534. doi: 10.1371/journal.pgen.1003534

Beldade P, Brakefield PM, Long AD (2002) Contribution of Distal-less to quantitative variation in butterfly eyespots. Nature 415:315-318. doi: $10.1038 / 415315 a$

Bento G, Ogawa A, Sommer RJ (2010) Co-option of the hormone-signalling module dafachronic acid-DAF-12 in nematode evolution. Nature 466:494-497. doi: 10.1038/nature09164

Bradshaw AD (1965) Evolutionary Significance of Phenotypic Plasticity in Plants. In: Thoday EWC and JM (ed) Advances in Genetics. Academic Press, pp 115-155

Brakefield PM, Gates J, Keys D, et al (1996) Development, plasticity and evolution of butterfly eyespot patterns. Nature 384:236-42.

Cheng LY, Bailey AP, Leevers SJ, et al (2011) Anaplastic lymphoma kinase spares organ growth during nutrient restriction in Drosophila. Cell 146:435-447. doi: $10.1016 /$ j.cell.2011.06.040 
Dembeck LM, Huang W, Magwire MM, et al (2015) Genetic Architecture of Abdominal Pigmentation in Drosophila melanogaster. PLoS Genet 11:e1005163. doi: 10.1371/journal.pgen.1005163

Dworkin I (2005) Towards a genetic architecture of cryptic genetic variation and genetic assimilation: the contribution of K. G. Bateman. J Genet 84:223226.

Endler L, Betancourt AJ, Nolte V, Schlötterer C (2015) Reconciling Differences in Pool-GWAS between Populations: A Case Study of Female Abdominal Pigmentation in Drosophila melanogaster. Genetics. doi: 10.1534/genetics.115.183376

Fedorka KM, Copeland EK, Winterhalter WE (2013) Seasonality influences cuticle melanization and immune defense in a cricket: support for a temperature-dependent immune investment hypothesis in insects. J Exp Biol 216:4005-10. doi: 10.1242/jeb.091538

Ghalambor CK, Hoke KL, Ruell EW, et al (2015) Non-adaptive plasticity potentiates rapid adaptive evolution of gene expression in nature. Nature 525:372-375. doi: 10.1038/nature15256

Gibert J-M, Mouchel-Vielh E, De Castro S, Peronnet F (2016) Phenotypic Plasticity through Transcriptional Regulation of the Evolutionary Hotspot Gene tan in Drosophila melanogaster. PLoS Genet 12:e1006218. doi: 10.1371/journal.pgen.1006218

Gibert J-M, Mouchel-Vielh E, Peronnet F (2017) Modulation of yellow expression contributes to thermal plasticity of female abdominal pigmentation in Drosophila melanogaster. Sci Rep 7:43370. doi: 10.1038/srep43370

Gibert P, Moreteau B, David JR (2000) Developmental constraints on an adaptive plasticity: reaction norms of pigmentation in adult segments of Drosophila melanogaster. Evol Dev 2:249-60.

Gibson G, Hogness DS (1996) Effect of polymorphism in the Drosophila regulatory gene Ultrabithorax on homeotic stability. Science 271:200-3.

Gilbert SF (2001) Ecological developmental biology: developmental biology meets the real world. Dev Biol 233:1-12. doi: 10.1006/dbio.2001.0210

James AC, Azevedo RB, Partridge L (1995) Cellular basis and developmental timing in a size cline of Drosophila melanogaster. Genetics 140:659-66.

Jeong S, Rebeiz M, Andolfatto P, et al (2008) The evolution of gene regulation underlies a morphological difference between two Drosophila sister species. Cell 132:783-93.

Johannsen W (1911) The genotype conception of heredity. Am Nat XLV:129-159. 
Kiontke K, Fitch DHA (2010) Phenotypic plasticity: different teeth for different feasts. Curr Biol CB 20:R710-712. doi: 10.1016/j.cub.2010.07.009

Kucharski R, Maleszka J, Foret S, Maleszka R (2008) Nutritional control of reproductive status in honeybees via DNA methylation. Science 319:1827-30.

Kutch IC, Sevgili H, Wittman T, Fedorka KM (2014) Thermoregulatory strategy may shape immune investment in Drosophila melanogaster. J Exp Biol 217:3664-9. doi: 10.1242/jeb.106294

Laland K, Uller T, Feldman M, et al (2014) Does evolutionary theory need a rethink? Nature 514:161-164. doi: 10.1038/514161a

Laland KN, Uller T, Feldman MW, et al (2015) The extended evolutionary synthesis: its structure, assumptions and predictions. Proc Biol Sci 282:20151019. doi: 10.1098/rspb.2015.1019

Leung A, Parks BW, Du J, et al (2014) Open chromatin profiling in mice livers reveals unique chromatin variations induced by high fat diet. J Biol Chem. doi: $10.1074 /$ jbc.M114.581439

Martin A, Orgogozo V (2013) The Loci of repeated evolution: a catalog of genetic hotspots of phenotypic variation. Evolution 67:1235-50. doi: 10.1111/evo.12081

Michie LJ, Mallard F, Majerus ME, Jiggins FM (2010) Melanic through nature or nurture: genetic polymorphism and phenotypic plasticity in Harmonia axyridis. J Evol Biol 23:1699-707. doi: 10.1111/j.14209101.2010.02043.x

Moczek AP, Sultan S, Foster S, et al (2011) The role of developmental plasticity in evolutionary innovation. Proc Biol Sci. doi: 10.1098/rspb.2011.0971

Monteiro A, Chen B, Ramos DM, et al (2013) Distal-less regulates eyespot patterns and melanization in Bicyclus butterflies. J Exp Zoolog B Mol Dev Evol 320:321-331. doi: 10.1002/jez.b.22503

Muschick M, Barluenga M, Salzburger W, Meyer A (2011) Adaptive phenotypic plasticity in the Midas cichlid fish pharyngeal jaw and its relevance in adaptive radiation. BMC Evol Biol 11:116. doi: 10.1186/1471-2148-11116

Pfennig DW, Wund MA, Snell-Rood EC, et al (2010) Phenotypic plasticity's impacts on diversification and speciation.

Pigliucci M (2001) Phenotypic Plasticity, Beyond Nature and Nurture. Baltimore and London

Prud'homme B, Gompel N, Carroll SB (2007) Emerging principles of regulatory evolution. Proc Natl Acad Sci U A 104 Suppl 1:8605-12. 
Ragsdale EJ, Müller MR, Rödelsperger C, Sommer RJ (2013) A developmental switch coupled to the evolution of plasticity acts through a sulfatase. Cell 155:922-933. doi: 10.1016/j.cell.2013.09.054

Rajpurohit S, Richardson R, Dean J, et al (2016) Pigmentation and fitness tradeoffs through the lens of artificial selection. Biol Lett. doi: $10.1098 / \mathrm{rsbl} .2016 .0625$

Scheiner SM (1993) Plasticity as a Selectable Trait: Reply to Via. Am Nat 142:371-373.

Schlichting CD, Pigliucci M (1993) Control of phenotypic plasticity via regulatory genes. Am Nat 142:366-370. doi: 10.1086/285543

Schmalhausen II (1949) Factors of Evolution, The Theory of Stabilizing Selection. The University of Chicago Press, Chicago and London

Schneider RF, Meyer A (2017) How plasticity, genetic assimilation and cryptic genetic variation may contribute to adaptive radiations. Mol Ecol 26:330350. doi: $10.1111 / \mathrm{mec} .13880$

Schwander T, Leimar O (2011) Genes as leaders and followers in evolution. Trends Ecol Evol 26:143-151. doi: 10.1016/j.tree.2010.12.010

Serobyan V, Xiao H, Namdeo S, et al (2016) Chromatin remodelling and antisense-mediated up-regulation of the developmental switch gene eud1 control predatory feeding plasticity. Nat Commun 7:12337. doi: $10.1038 /$ ncomms12337

Shirai LT, Saenko SV, Keller RA, et al (2012) Evolutionary history of the recruitment of conserved developmental genes in association to the formation and diversification of a novel trait. BMC Evol Biol 12:21. doi: 10.1186/1471-2148-12-21

Sicard A, Thamm A, Marona C, et al (2014) Repeated evolutionary changes of leaf morphology caused by mutations to a homeobox gene. Curr Biol 24:18806. doi: 10.1016/j.cub.2014.06.061

Simola DF, Graham RJ, Brady CM, et al (2016) Epigenetic (re)programming of caste-specific behavior in the ant Camponotus floridanus. Science. doi: $10.1126 /$ science.aac6633

Susoy V, Ragsdale EJ, Kanzaki N, Sommer RJ (2015) Rapid diversification associated with a macroevolutionary pulse of developmental plasticity. eLife. doi: 10.7554/eLife.05463

Suzuki Y, Nijhout HF (2006) Evolution of a polyphenism by genetic accommodation. Science 311:650-2. 
Tang HY, Smith-Caldas MS, Driscoll MV, et al (2011) FOXO regulates organspecific phenotypic plasticity in Drosophila. PLoS Genet 7:e1002373. doi: 10.1371/journal.pgen.1002373

True JR, Yeh SD, Hovemann BT, et al (2005) Drosophila tan Encodes a Novel Hydrolase Required in Pigmentation and Vision. PLoS Genet 1:e63.

Via S (1993) Adaptive phenotypic plasticity: target or by-product of selection in a variable environment? Am Nat 142:352-365. doi: 10.1086/285542

Via S, Gomulkievicz R, de Jong G, et al (1995) Adaptive phenotypic plasticity: consensus and controversy. TREE 10:212-217.

Waddington CH (1952) Selection of the genetic basis for an acquired character. Nature 169:278.

Waddington CH (1956) Genetic Assimilation of the Bithorax Phenotype. Evolution 10:1-13. doi: 10.2307/2406091

Waddington CH (1959) Canalization of development and genetic assimilation of acquired characters. Nature 183:1654-5.

West-Eberhard MJ (2003) Developmental plasticity and evolution. Oxford University Press, New York

West-Eberhard MJ (2005) Developmental plasticity and the origin of species differences. Proc Natl Acad Sci U A 102 Suppl 1:6543-9.

Whitehead A, Roach JL, Zhang S, Galvez F (2011) Genomic mechanisms of evolved physiological plasticity in killifish distributed along an environmental salinity gradient. Proc Natl Acad Sci U A 108:6193-8. doi: 10.1073/pnas.1017542108

Whitehead A, Roach JL, Zhang S, Galvez F (2012) Salinity- and populationdependent genome regulatory response during osmotic acclimation in the killifish (Fundulus heteroclitus) gill. J Exp Biol 215:1293-1305. doi: 10.1242/jeb.062075

Wittkopp PJ, Beldade P (2009) Development and evolution of insect pigmentation: genetic mechanisms and the potential consequences of pleiotropy. Semin Cell Dev Biol 20:65-71.

Wittkopp PJ, Stewart EE, Arnold LL, et al (2009) Intraspecific polymorphism to interspecific divergence: genetics of pigmentation in Drosophila. Science 326:540-4. doi: 10.1126/science. 1176980

Wittkopp PJ, Williams BL, Selegue JE, Carroll SB (2003) Drosophila pigmentation evolution: divergent genotypes underlying convergent phenotypes. Proc Natl Acad Sci U A 100:1808-13. 
Woltereck R (1909) Weitere experimentelle unters"uchungen "uber artver"anderung, speziell "uber das wesen quantitativer artunterschiede bei daphniden. Verhandlungen Dtsch. Zooligischen Ges. 110-172.

652

653

654

655

656

657

658

659
Wund MA, Baker JA, Clancy B, et al (2008) A test of the "flexible stem" model of evolution: ancestral plasticity, genetic accommodation, and morphological divergence in the threespine stickleback radiation. Am Nat 172:449-462. doi: $10.1086 / 590966$

Yassin A, Bastide H, Chung H, et al (2016) Ancient balancing selection at tan underlies female colour dimorphism in Drosophila erecta. Nat Commun 7:10400. doi: 10.1038/ncomms10400 
662 Figure 1: Above: wild-type Drosophila melanogaster female. Bellow: female

663 Drosophila showing a bithorax phenotype obtained after genetic assimilation of 664 the bithorax phenocopy initially induced by ether treatment of the embryo 665 (modified after (Waddington 1956)). The post-notum is replaced by an 666 additional meso-notum and halteres are replaced by rudimentary wings.

667

668 Figure 2: Left: the network of rivers flowing into the Chesapeake-bay, in north669 east America, constitutes a range of water habitat with different salinity going 670 from pure sea water (dark blue) to pure fresh water (red), modified after 671 (Whitehead et al. 2011). Top right, populations of Atlantic killifish (Fundulus 672 heteroclitus) are locally adapted to particular salinities but are also able to 673 quickly adjust to osmotic challenges. Bottom right: modification of gill 674 epithelium after transfer form sea water to fresh water (after (Whitehead et al. 675 2012)). In sea water (32 ppt), the apical surface of the gill epithelium is made of 676 pavement cells (red asterisk) interspersed with mitochondrion-rich cells 677 possessing apical crypt with small surface area (green arrowhead). Exposure to 678 fresh water ( $0.1 \mathrm{ppt})$ leads to an enlargement of the apical surface of 679 mitochondrion-rich cells (green arrowhead).

681 Figure 3: Top: natural populations of the satyrine butterfly Bicyclus anynana 682 show two seasonal morphs, the dry season morph (left) and the wet season 683 morph (right) that can be produced in the lab by rearing the larvae at $17^{\circ} \mathrm{C}$ or $68427^{\circ} \mathrm{C}$, respectively. Middle and bottom: the dry season and the wet season 
685 morphs can be fixed by artificial selection leading to a LOW line (middle) or a 686 HIGH line (bottom) with very limited plasticity (after (Brakefield et al. 1996)).

688 Figure 4: The diplogastrid nematod Pristionchus pacificus displays two 689 alternative tooth morphs: a wide one, eurystomatous (Eu, left) or a narrow one 690 stenostomatous (St, right), that are produced after an irreversible decision 691 during larval development (after (Kiontke and Fitch 2010)). The ratio of these 692 two morphs is variable between strains and is modulated by environmental 693 factors such as crowding and starvation.

694

695 Figure 5: Capsella grandiflora and Capsella rubella differ regarding the 696 dissection of their leaf margins. This difference was mapped to the RCO-A locus. 697 Above nearly isogenic lines (NIL) differing only in the $R C O-A$ locus region. The 698 line with the C. grandiflora allele (NILgg) (left) does not show any leaf margin 699 dissection at $22^{\circ} \mathrm{C}$ contrary to the line with the C. rubella allele (NILrr) (right). 700 Lower temperature $\left(16^{\circ} \mathrm{C}\right)$ induces $(N I L g g)$ or increases (NILrr) leaf margin 701 dissection.

703 Figure 6: Abdominal cuticles of females Drosophila melanogaster from an inbred 704 line $\left(w^{1118}\right)$ grown at $18^{\circ} \mathrm{C}$ (left) or $29^{\circ} \mathrm{C}$ (right). Female abdominal pigmentation 705 is sensitive to temperature during development in particular in the most 706 posterior segments (A5, A6 and A7). Anterior is up, ventral is left (after (Gibert 707 et al. 2016)). 

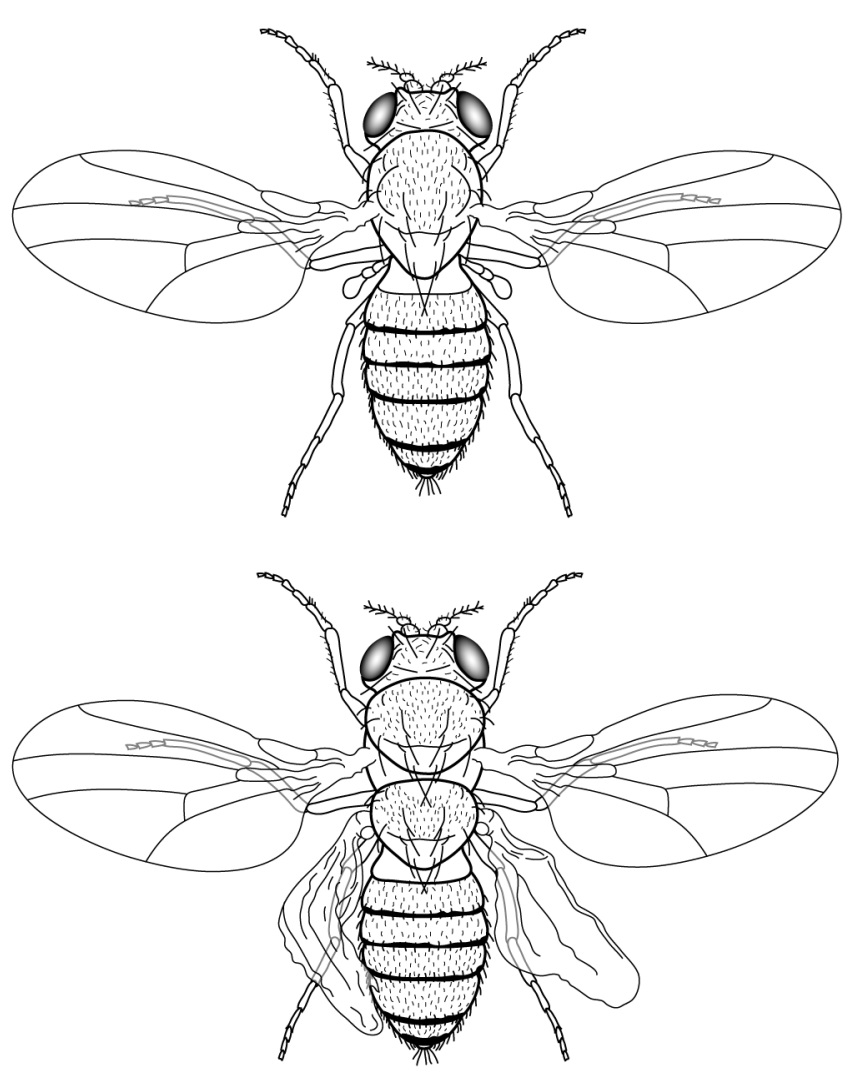

709 


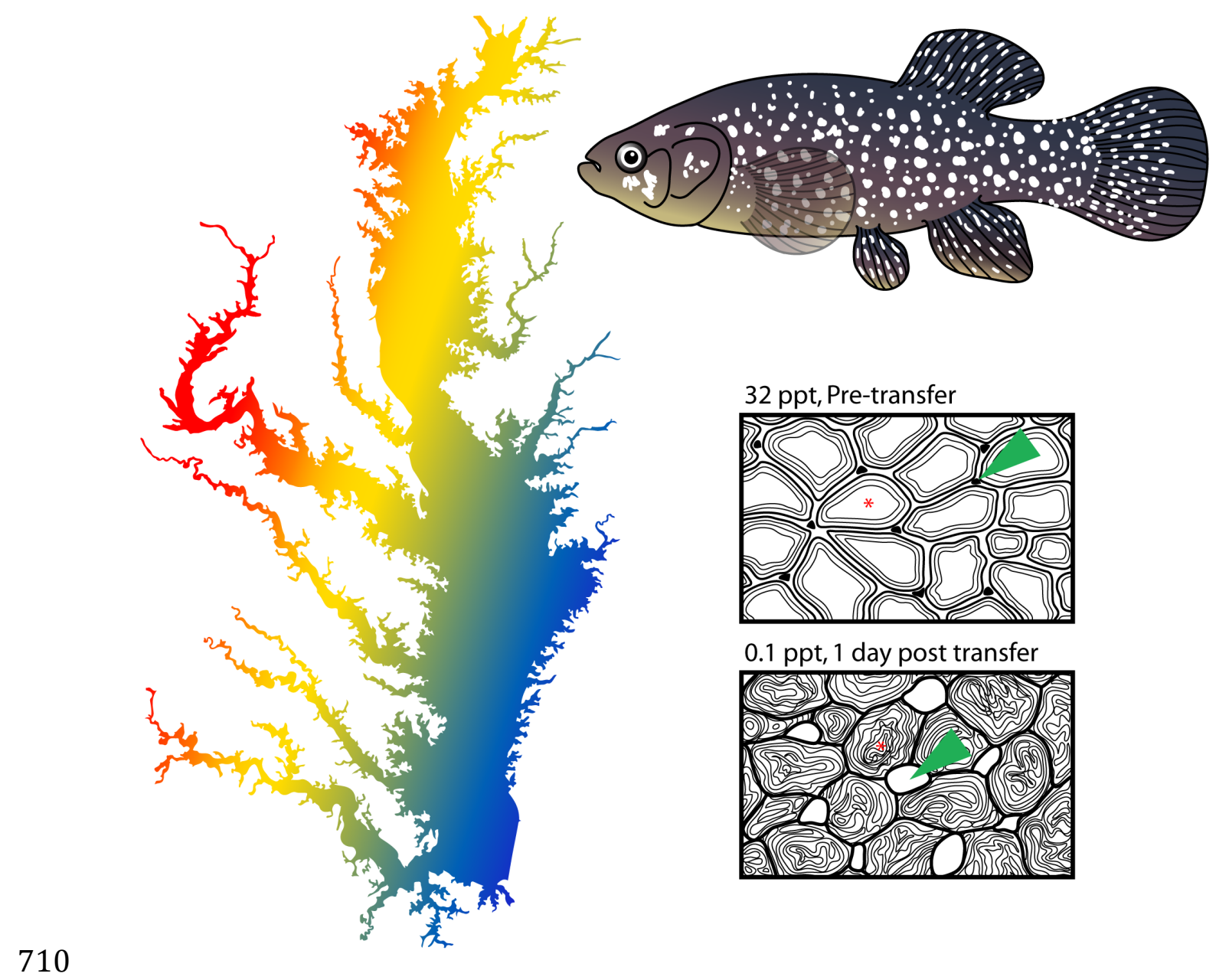

711 


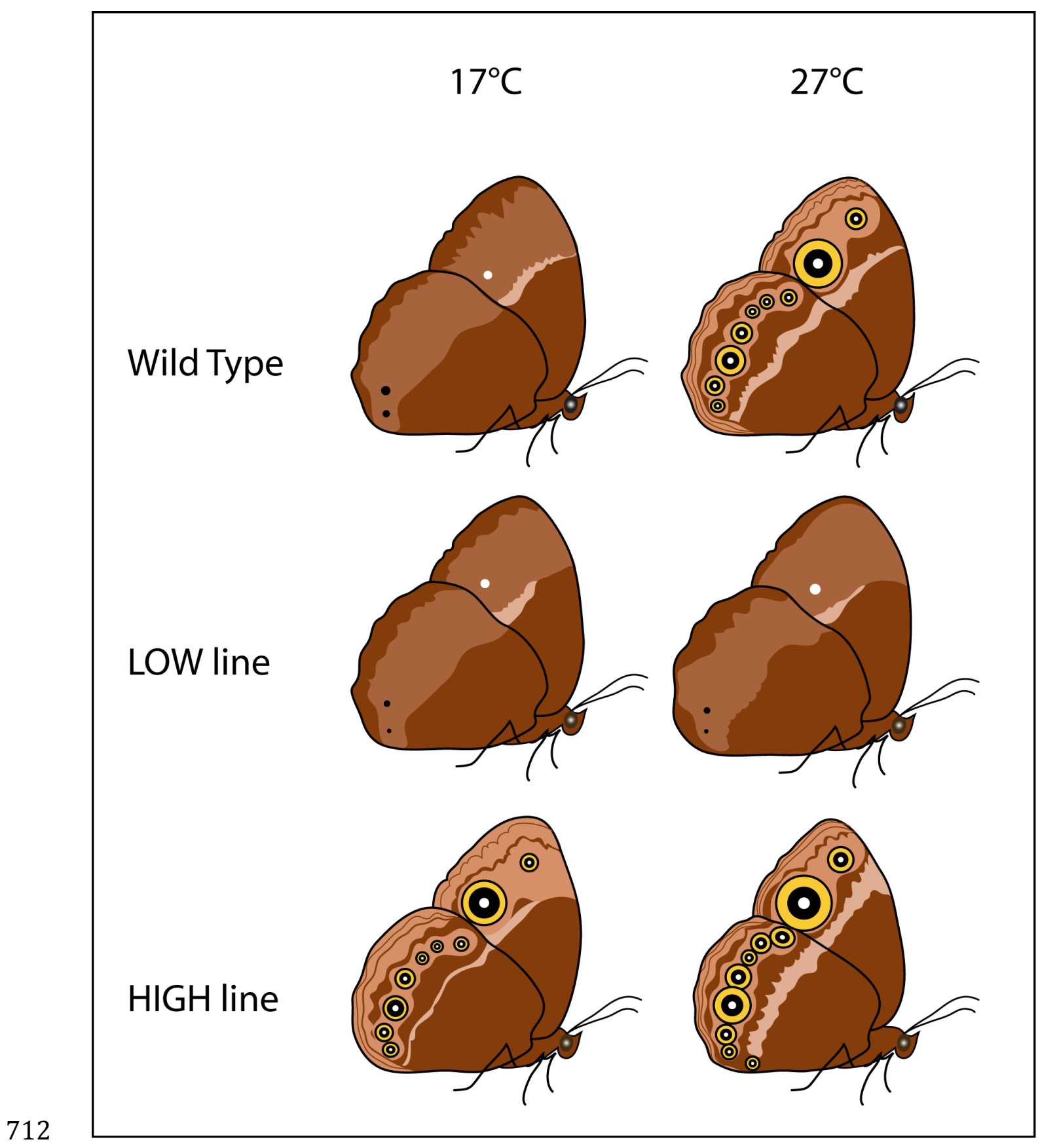

713 


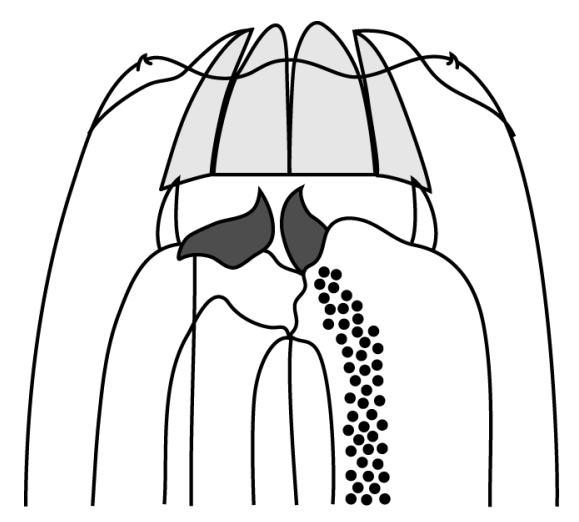

714 Eurystomatous (Eu)

715

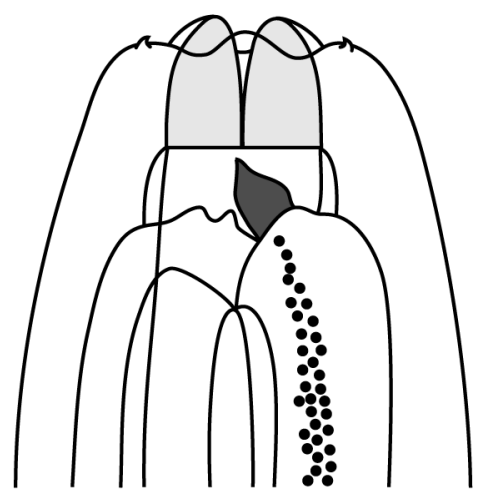

Stenostomatous (St)
NILgg
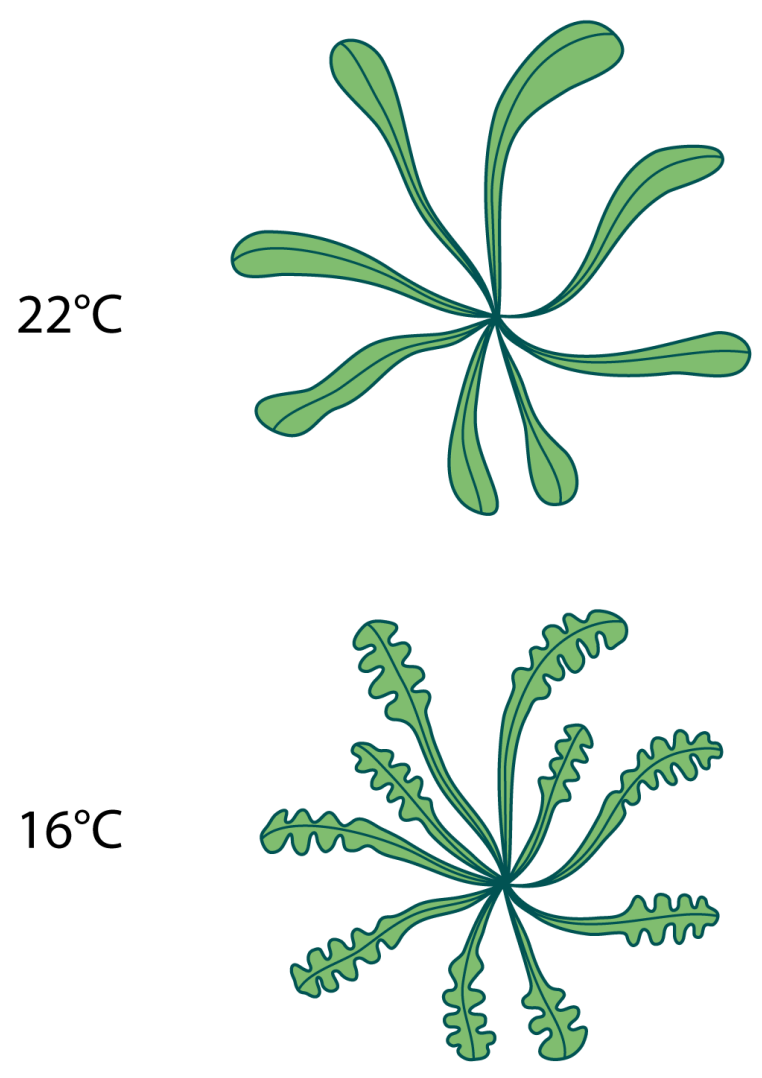

NILrr
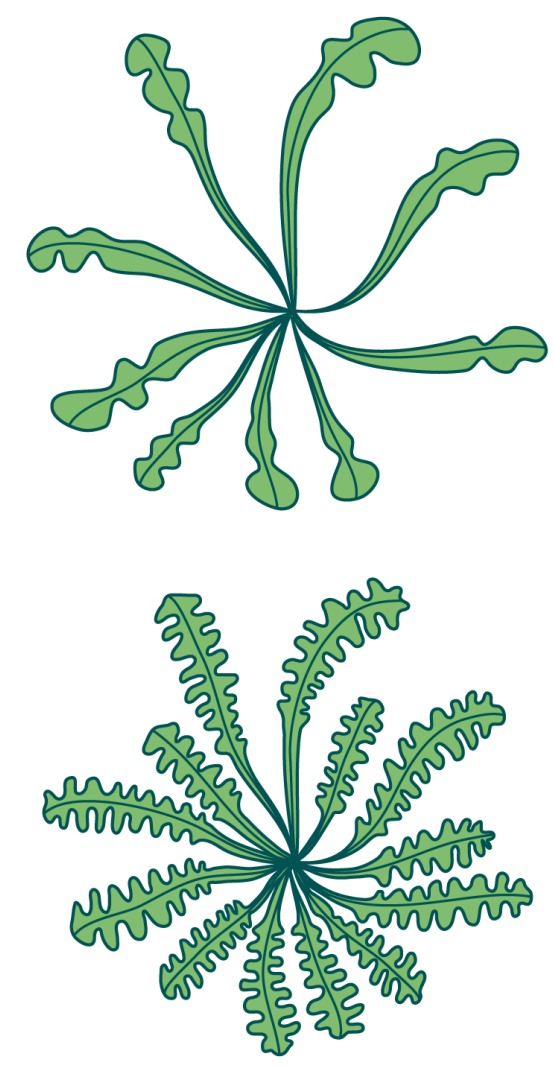

716 


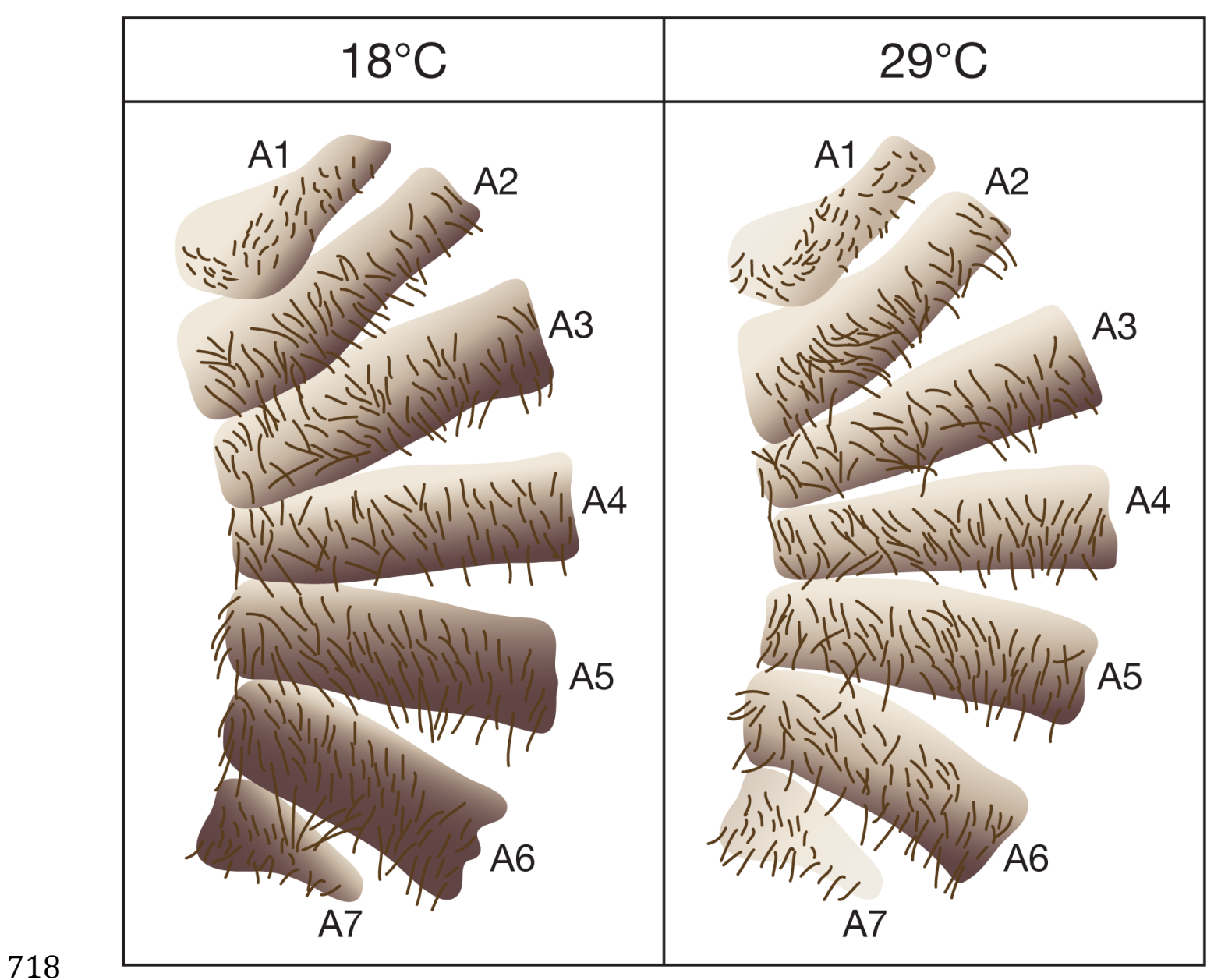

719

720 